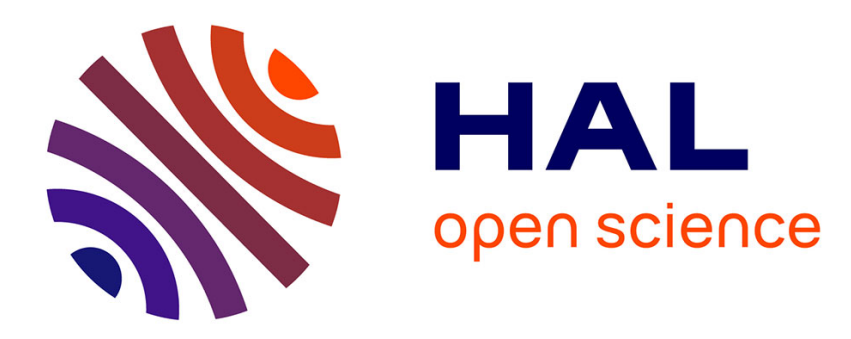

\title{
Réalité et discursivité. D'autres dimensions pour la théorie du discours
}

\author{
Marie-Anne Paveau
}

\section{To cite this version:}

Marie-Anne Paveau. Réalité et discursivité. D'autres dimensions pour la théorie du discours. Semen

- Revue de sémio-linguistique des textes et discours, 2012, 34, pp.95-115. hal-00773187

\section{HAL Id: hal-00773187 \\ https://hal.science/hal-00773187}

Submitted on 11 Jan 2013

HAL is a multi-disciplinary open access archive for the deposit and dissemination of scientific research documents, whether they are published or not. The documents may come from teaching and research institutions in France or abroad, or from public or private research centers.
L'archive ouverte pluridisciplinaire HAL, est destinée au dépôt et à la diffusion de documents scientifiques de niveau recherche, publiés ou non, émanant des établissements d'enseignement et de recherche français ou étrangers, des laboratoires publics ou privés. 


\section{Réalité et discursivité. D'autres dimensions pour la théorie du discours}

\section{Introduction ${ }^{1}$}

L'analyse du discours entretient depuis ses fondations un rapport distant et complexe à la réalité. Les courants les plus fidèles à la linguistique saussurienne maintiennent le primat de la langue et perpétuent un constructivisme sémantique qui met en son centre un ordre de la langue ou du discours irréductible à un ordre de la réalité. Les sensibilités plus « sociales » prennent en compte la réalité sous les notions de contexte, d'extralinguistique ou d'extérieurs du discours. Ces positions ont en commun de considérer la réalité comme une donnée secondaire de la construction des discours (Achard-Bayle 2006 (éd.), Achard-Bayle, Paveau 2007 (éds)).

Or, certaines propositions faites dès les débuts des analyses du discours vers 1960-70 évoquent pourtant précisément une coconstitution des discours et des données contextuelles, comme le précise ce célèbre extrait de « La sémantique et la coupure saussurienne »: «[...] le lien qui relie les "significations" d'un texte aux conditions socio-historiques de ce texte n'est nullement secondaire, mais constitutif des significations elles-mêmes " (Pêcheux et al. 1971 in Maldidier 1990 : 141).

Mais, faute sans doute d'un appareillage théorique adéquat et d'une configuration épistémologique qui auraient permis à la théorie du discours de penser la réalité au-delà de la question de la référence, cette dimension réaliste n'a pas vraiment été envisagée.

Les nouvelles formes de réalisme proposées ces vingt dernières années peuvent contribuer de manière intéressante au renouvèlement du regard de l'analyse des discours. II s'agit en particulier du réalisme naturel tel que le propose Putnam $(1990,1999)$ et du réalisme moral très discuté en philosophie morale (Virvidakis 1994, Ogien (dir.) 1999). Par ailleurs, les développements en philosophie de l'esprit sur la nature de la conscience ont fait émerger une perspective externaliste (Clark \& Chalmers 1998, Dennett 1993 [1991], Brassac (dir.) 2006) qui permet de dépasser le binarisme esprit/monde et de montrer comment les productions langagières sont coconstruites dans et par les environnements. Dans les deux cas, on repense les dualismes traditionnels de la pensée occidentale ordonnés par la macro-paire mind/body ${ }^{2}$ et, tout particulièrement, en ce qui concerne la linguistique, le dualisme langue/monde ou linguistique/extralinguistique.

Après avoir exposé les données postdualistes mobilisables dans une théorie linguistique du discours, j'essaierai de montrer comment ces réflexions peuvent permettre de modifier et d'enrichir les notions de sujet, de contexte et de langue. Je développerai enfin les deux exemples de la dimension matérielle des discours (les technologies discursives) et de leur dimension morale (la notion de vertu discursive).

\section{Des approches postdualistes pour penser la réalité}

On a assisté dans les deux dernières décennies, en sciences humaines et sociales, et tout particulièrement en philosophie, anthropologie, sociologie et sciences de la cognition, à une évolution épistémologique qui peut modifier les perceptions des catégories intellectuelles ayant permis jusqu'à présent de penser le monde et d'élaborer les disciplines.

\subsection{La remise en cause des grands dualismes}

Les grands dualismes (esprit/monde, intellect/affect, fait/valeur, homme/machine, homme/animal, etc.) sont retravaillés et remis en cause (Schaeffer 2007) au profit de visions plus complexes qui décrivent des catégories mixtes voire hybrides : les conceptions externalistes de l'esprit présentent ce dernier comme distribué dans les environnements, qui assument le rôle

\footnotetext{
${ }^{1}$ Cet article applique les rectifications orthographiques proposées par l'arrêté de décembre 1990, y compris dans les citations.

${ }^{2}$ Sur la place du mind body problem dans les linguistiques cognitives, voir Achard-Bayle, Paveau 2012.
} 
d'agents psychiques externes (Hutchins $1995^{3}$ ) ; le rôle des émotions est désormais intégré aux activités de la raison (Damasio 2005 [2003], Plantin 2011) ; on a décrit l'imprégnation axiologique de phénomènes pourtant perçus comme «objectifs» (Putnam 2004 [2002]) ; les couplages homme-machine, homme-animal et même homme-animal-machine sont devenus des objets de réflexion importants (Latour 2005, Chapouthier, Kaplan 2011). II me semble que la linguistique dans l'ensemble de ses versions, et particulièrement la théorie du discours, ne peut qu'être prise dans ces évolutions. Avec Schaeffer, j'accepte "la fin de l'exception humaine ", c'est-à-dire la remise en cause de l'idée que l'être humain transcenderait sa nature biologique, en particulier par des propriétés sociale et culturelle qui lui seraient spécifiques et désarrimées de la nature. Cela implique de se situer dans un cadre naturaliste, qui n'est pas forcément réductionniste :

La biologie de l'évolution implique en effet une naturalisation de l'identité humaine. Pour éviter tout malentendu, je précise que dans le présent contexte ce terme de «naturalisation " signifie simplement que l'identité de l'homme est pensée comme étant celle d'une forme de vie biologique - rien de moins et rien de plus. Les conséquences essentielles de cette naturalisation sont au nombre de quatre.

- D'abord, dans la perspective de la biologie de l'évolution l'homme n'apparait pas simplement comme un être qui a un aspect biologique [...], mais il est un être biologique [...]

- En deuxième lieu, elle implique une historisation de l'identité humaine [...].

- En troisième lieu, cette perspective naturaliste est foncièrement non essentialiste [...].

- Enfin [...] il s'agit d'une conception non finaliste [...]

(Schaeffer 2007 : 62-63; soulignements de l'auteur)

Cette conception m'amène à une conception écologique de la vie humaine et, partant, de la production langagière qui est l'une des principales activités humaines : produire des discours ne sera plus l'apanage de l'être humain possédant un statut exceptionnel et supérieur dans la nature, mais sera la manifestation de son comportement d'être vivant dans ses environnements, au même titre que les autres.

\subsection{L'externalisme au sens large}

Dès le début du $20^{\mathrm{e}}$ siècle, la cognition est pensée comme un système non seulement interhumain mais également environnemental, par certains philosophes, anthropologues ou linguistes : chez Bakhtine-Volochinov, Malinovski, Sapir, et aussi Vygotski, Peirce, Wittgenstein, le contexte est un élément essentiel à la compréhension de la pensée et du langage. À partir des années 1990, la cognition emprunte un «tournant social » (plus précisément, social et culturel), appuyé sur l'hypothèse externaliste.

\section{L'extended mind}

L'hypothèse externaliste permet de " décloisonner » l'esprit, qui constitue le lieu de la cognition pour le courant cognitiviste dit orthodoxe. Mais, pour comprendre l'externalisme, il faut définir clairement l'opposition interne vs externe. On peut lui donner, comme le précise Lenay 2006, un sens « faible », qui a trait au « contenu intentionnel des représentations ou états mentaux ». Alors que l'internalisme considère que ce contenu est issu des relations internes entre les représentations, l'externalisme y ajoute les "relations causales entre organisme et l'environnement » (Lenay, $2006:$ 47). La version dite « forte », dont Hutchins 1995 ou Clark 1997 ou encore Clark \& Chalmers 1998 et Sinha 1988 sont de bons représentants, considère que « les dispositifs techniques ne réalisent pas seulement des modifications artificielles d'une cognition qui serait "naturelle", mais ont un rôle constitutif des activités cognitives pour la perception, le raisonnement, la mémoire, l'imagination ou les interactions " (Lenay, 2006 : 47). II faut noter l'emploi du terme constitutif, certes différent de celui souligné plus haut chez Pêcheux et al. 1971 , mais qui insiste cependant sur cette même idée d'assemblage ou de composition (au sens de création d'un composite) dans lequel les différents matériaux ne sont pas séparables. "Ainsi,

\footnotetext{
${ }^{3}$ Le cadre théorique de la cognition distribuée a été principalement élaboré par Hutchins et ses collègues à San Diego (Hutchins 1994 [1991], 1995). Le but est de dépasser le niveau d'analyse traditionnel en sciences cognitives (l'individu) pour caractériser une cognition située et incarnée (embodied) dans son contexte d'occurrence: la cognition est distribuée entre agents et éléments de la situation. Dans son étude de l'activité de pilotage dans un avion (1991), Hutchins délaisse le niveau traditionnel d'analyse (les processus individuels de représentation et de traitement par le pilote et le copilote) pour adopter le cockpit comme unité d'analyse pertinente. Le chercheur analyse alors les propriétés cognitives du cockpit et non pas celles des individus présents dans le cockpit.
} 
dans le cadre de la cognition située, continue Lenay, l'activité cognitive d'un ou plusieurs sujets peut se comprendre par un jeu d'inscriptions dans le milieu et d'interprétations perceptives [...] de l'organisation spatiale d'objets et de symboles. [...] Les inscriptions servent de mémoire externe et partageable [...] » (2006: 47). C'est cette version forte que j'adopte, mais sans renoncer aux représentations internes: dans la cognition externaliste que je défends, l'extérieur et l'extérieur sont assemblés et forment un continuum, sans être opposés.

\section{La cognition sociale}

Cette étiquette est préférable à celle de constructivisme, qui a le désavantage de permettre une opposition binaire qui me semble réductrice pour les sciences cognitives : constructivisme vs internalisme ou même parfois constructivisme vs cognitivisme. La cognition sociale rassemble de nombreux courants en anthropologie et sociologie (Lave, Suchman, Hutchins ou Latour pour le monde anglophone, Latour également à partir des années 1990, Quéré, Conein pour la France). L'idée d'une cognition sociale avec une base externaliste s'est également développée en philosophie (Clark, Dennett, Livet), en psychologie (Sinha), mais aussi dans les domaines du design (Norman) et de l'écologie (Gibson). La cognition sociale fédère des approches différentes comme la cognition située, partagée, incarnée, distribuée. Je ne les détaille pas mais je résume les thèses du champ, dont les implications linguistiques seront détaillées plus bas :

- les activités cognitives ne sont pas ou pas seulement déterminées par des représentations internes individuelles; l'activité cognitive trouve dans l'environnement physique, culturel, historique, etc. différents types de supports matériels ou immatériels, naturels ou artefactuels.

- les données cognitives (informations, savoirs, croyances, etc.) se propagent dans l'ensemble du système cognitif constitué par les individus et les agents non humains de l'environnement.

- le contrôle des activités cognitives n'est pas centralisé mais " partagé 》 ou "distribué " entre les différents agents cognitifs.

Dans un article de synthèse sur le constructivisme, écrit dans le cadre des " sciences de la culture " lancées par Rastier (Bouquet, Rastier dir. 2002), Bischofsberger décrit ainsi ce qu'il appelle « la lignée socioculturelle » des sciences cognitives :

Quant à la lignée socioculturelle, elle fait de la cognition un phénomène discursif, situé dans des contextes historiques et sociaux. La cognition quitte, pour ainsi dire, la " tête » de l'individu, pour devenir un phénomène social et distribué, émergeant dans des conditions sociales et culturelles contingentes. Ce courant repose sur la conviction que toute connaissance humaine est le produit d'un acte interprétatif conditionné par des présupposés sociaux (Bischofsberger, 2002 : 167).

Si l'on considère en effet que l'activité de langage est une activité cognitive, alors on voit les implications de cette conception : la production langagière ne peut plus être observée à partir d'un seul point de vue interne, mais on doit construire un point de vue écologique, qui permet de la décrire au sein de son environnement.

\section{La théorie du discours réinterrogée}

J'emprunte le terme symétrique à Latour qui propose en 1997 son "anthropologie symétrique ", c'est-à-dire une anthropologie qui ne considère plus le rapport entre l'humain et le non-humain comme une frontière mais comme un continuum : l'intersubjectivité qui fonde le social chez les sujets humains est aussi une « interobjectivité » (1994), puisque les objets, l'ensemble du monde non humain, contribuent à la définition du social. Latour propose donc une sociologie alternative, qui prend en compte les « matières " hétérogènes qui font assemblage dans le social, et conteste l'idée d'un social homogène: on ne peut faire de la sociologie avec du social uniquement, mais il faut tenir compte de la nature « assemblée » des phénomènes sociaux. De la même manière, il me semble que la linguistique, et en particulier la théorie du discours, pourrait étendre la définition du langagier en s'enrichissant de matérialités sociales, culturelles, environnementales, objectales, etc.

\subsection{Une approche symétrique}

Les analyses du discours qui se pratiquent en France depuis une cinquantaine d'années reposent sur une approche dualiste, distinguant le contexte comme extérieur au discours (l'extralinguistique ou extradiscursif). De ce fait, elles sont logocentrées (les observables sont de 
nature «purement » langagière) voire egocéphalocentrées ${ }^{4}$ (les observables sont construits à partir des locuteurs, considérés comme des sujets distincts et séparables des environnements). Cela veut dire que le projet de l'intégration constitutive du contexte à la théorie du discours n'a pas réellement abouti, le contexte (les « conditions de production» dans le vocabulaire de la théorie du discours marxo-freudienne) restant de l'ordre de l'arrière-plan.

Une approche symétrique postulerait que les unités dites «non linguistiques » ou « extralinguistiques » participent pleinement à l'élaboration de la production verbale, au sein d'un continuum entre verbal et non verbal, et non plus une opposition (Paveau 2009). Ce point de vue n'est cependant pas révolutionnaire, puisque certains auteurs, dans des domaines liés aux sciences du langage, ont proposé antérieurement des perspectives de ce type. Mais comme le soulignent Brassac et al. 2008a, cela reste une pétition de principe : « [...] although the ecology of action is becoming a key topic in various disciplines, studies that explicitly take artifacts and gestures into account - both theoretically and empirically - as they are actually mobilized by participants in interaction have remained underdeveloped » (2008a : 208).

L'interactionnisme et la psychologie sociocognitive constituent de bons exemples de ce que l'on peut appeler travaux "symétriques". Les interactionnistes proposent en effet depuis longtemps d'intégrer les éléments de l'environnement dans l'analyse linguistique. Dès ses Éléments de pragmatique linguistique en 1981, Berrendonner propose de prendre en compte l'aspect signifiant de tous les éléments de la réalité via la notion de symptôme. Les chercheurs de l'école de Genève, autour de Bronckart et du courant de l'interactionnisme sociodiscursif, pratiquent aussi cette linguistique «ouverte » sur ses extérieurs, à partir des propositions de Vygotski et l'école russe de la théorie de l'activité de années 1920 en psychologie. II faudrait aussi mentionner l'analyse conversationnelle issue de l'ethnométhodologie (Schegloff, Sacks, puis Mondada par exemple), qui développe des approches multimodales. Mais, dans ces travaux, sur le plan linguistique, l'environnement matériel semble encore réduit au corps et aux gestes " non verbaux ", comme le montre le récent bilan Pirstec: "L'acte moteur doit être vu non pas uniquement du point de vue de l'acteur, mais également de celui de l'observateur qui cherche à relier geste et sémantique, gestes et communication en contexte social. II s'agit ici de comprendre le rôle de la motricité dans la communication sociale »(Atelier Pirstec 2010: 17 ; je souligne). Dans ce passage, on voit bien que «l'extralinguistique » ou le «non-verbal » est identifié et circonscrit au geste corporel. Si le sujet n'est plus "égocéphalocentré ", il reste cependant " corpocentré » et la frontière corps-monde est maintenue. Chez les psychologues sociaux qui s'intéressent à la cognition, et qui travaillent en interdisciplinarité, le corps est considéré comme un élément de l'environnement et c'est l'ensemble du système informationnel qui est constitué en observable. Dans le champ français, Brassac plaide activement pour une analyse située et véritablement interdisciplinaire des phénomènes langagiers. Deux études illustrent cette approche : Brassac et al. 2008a, étude des interactions dans un hôpital autour de l'informatisation d'un service, intégrant pleinement le rôle des objets et Brassac et al. 2008b, analyse de l'émergence de connaissances au cours d'un processus collectif rassemblant informaticiens et agronomes.

Dans le champ anglo-saxon, c'est Sinha qui me semble un des meilleurs représentants de cette conception (Sinha 2000, 2008) : son travail sur l'intersubjectivité, et la manière dont il propose d'intégrer les matérialités environnementales à la production du sens contribuent à ce décloisonnement des agents en jeu dans les activités cognitives, en particulier langagières.

\subsection{Enjeux linguistiques d'une cognition externe}

Le choix d'une conception externaliste implique d'observer sous un autre angle les notions constitutives de la linguistique déjà remises en jeu par la théorie du discours : le sujet, le contexte et la langue.

\section{La question du sujet}

\footnotetext{
${ }^{4}$ Brassac emprunte le mot à Kaufmann dans Ego. Pour une sociologie de l'individu. Une autre vision de l'homme et de la construction du sujet (Nathan, 2001) : " II est vrai qu'une grande partie des études conduites en psychologie sont largement ancrées dans un égocéphalocentrisme qui plombe, selon certains [Kaufmann 2001], leur capacité à appréhender la réelle nature des processus en jeu dans l'être-au-monde du sujet humain. Ainsi les solipsisme et mentalisme qui marquent par exemple la psychologie de la cognition laissent-ils en filigrane la question du rapport entre l'entité "pensante" et le monde "pensé"» (Brassac 2007 : 159).
} 
Dans La raison, le langage et les normes, Auroux mentionnait les «structures cognitives externes " qui nécessitent de penser la cognition linguistique en relation avec les réalités extérieures (Auroux 1998). Ces structures peuvent être des «outils linguistiques » (grammaires, dictionnaires) mais également des artefacts et des outils non « linguistiques ", qui contribuent cependant à la production langagière : instruments d'écriture, appareils de mesure (voir l'analyse du cockpit dans Hutchins 1994), ou même machine à café (voir Lahlou 2000a et b et son travail sur les « attracteurs cognitifs» et le travail de bureau). La production langagière n'est donc pas directement «interhumaine » mais médiée et distribuée. Des réflexions en anthropologie et philosophie sur la notion d'identité s'articulent avec l'approche externaliste et peuvent fournir un cadre épistémologique pour (re)penser le sujet. La réflexion de Laplantine dans Je, nous et les autres est absolument adéquate aux conceptions externalistes (Laplantine 2010 [1999]). Même si le concept est critique dans son esprit, et si sa position me semble conservatrice et déploratoire, la notion de «liquidité » avancée par Bauman aide cependant à penser les circulations et les décloisonnements postcartésiens $(2004,2007)$. Mais c'est sans doute la position d'Honneth qui est la plus opératoire en sciences humaines, car issue d'une synthèse puissante des remises en cause du sujet dans la seconde moitié du 20e siècle (Honneth 2008 [1993]). Entre deux conceptions extrêmes du sujet (sujet plein de la volonté et de la maitrise vs sujet vidé par les déterminismes), le philosophe propose la notion d'" autonomie décentrée ", ne souhaitant pas abandonner totalement le sujet au néant de ses assujettissements, mais ne visant pas non plus le retour à une conception préstructuraliste d'un moi de la maitrise, de la conscience et de la volonté.

On le voit, les circulations sont intenses entre les différents paradigmes qui s'occupent de la question du sujet dans une perspective, si ce n'est explicitement externaliste, du moins alternative au binarisme traditionnel. II ne me semble désormais plus possible d'adopter une conception egocentrée du sujet, qui détiendrait une perspective sur l'autre et sur le monde. Reste à assumer cette modification épistémologique dans les disciplines de la langue et du discours.

\section{La question du contexte}

II existe des synthèses récentes sur la question du contexte et les problèmes qu'elle pose aux différentes linguistiques : un numéro de Pratiques est consacré à cette question (Achard-Bayle (éd.) 2006), ainsi qu'un numéro de Corela qui étend la problématique textuelle et discursive aux approches cognitives (Achard-Bayle, Paveau (éd.) 2007). Dans Pratiques, on peut lire un article de Moirand défendant une conception dynamique, calée sur la progression de la recherche et donc sur une certaine forme de prise en compte de l'ensemble de l'environnement :

Actuellement, [...] je me situe dans une conception dynamique du contexte, ce qui, pour le chercheur en analyse du discours qui travaille sur les données empiriques, repose sur le repérage et la prise en compte d'indices de contextualisation de niveaux divers au fur et à mesure de l'avancée du recueil des données et des analyses effectuées (mais pas forcément dans l'ordre de l'énumération qui suit) :

- le cotexte linguistique, le cotexte linéaire du « fil » intratextuel (le fil horizontal du discours ou le fil déroulant de l'interaction et les reprises de formes diverses qui s'y manifestent) ;

- le contexte sémiotique et situationnel (le «hic» et «nunc» de la situation de communication) ;

- le contexte des séries génériques dans lesquelles s'inscrivent les unités discursives empiriques recueillies à l'intérieur d'un domaine, dans une situation de communication ou sur un type de support ;

- et enfin le contexte socio-historique qui entre de manière exhibée ou cachée dans les configuration discursives, donc tout ce qui relève de l'intertexte et de l'interdiscours (voire de I'histoire conversationnelle), ainsi que des mémoires collectives et des savoirs partagés, c'est-àdire de l'histoire... (Moirand $2006: 43$ ).

Les notions de "contexte des séries génériques », qui s'inscrit dans la problématique des genres de discours de nature sociocognitive pour Moirand et celle de " contexte socio-historique » s'inscrivant dans l'idée d'une mémoire interdiscursive, que la chercheuse a élaborée à partir de la notion de mémoire discursive proposée par Courtine, signalent en effet une prise en compte de la réalité un peu plus large et réaliste, que le contexte situationnel ou communicationnel. Mais cette conception me semble encore insuffisante pour refléter une véritable approche non dualiste ou postdualiste. Je prendrai deux exemples d'éléments manquants à ces conceptions du contexte : la dimension morale et la dimension technologique (traitée dans le paragraphe suivant). 
Une position intégrative et non dualiste par rapport aux catégories de l'activité humaine pose de fait un continuum entre le langagier et le non- ou extralangagier, ce dernier étant constitué par tous les paramètres qui constituent la réalité : aux deux éléments social et historique, qui composent le "socio-historique » de la théorie du discours, on peut donc naturellement ajouter par exemple le culturel (l'articulation langage-culture est ancienne, on le sait, et même antérieure à la fondation de la linguistique comme science du langage), l'émotionnel (ces quinze dernières années, les travaux sur langage et émotion se sont fortement développés) ou le moral ${ }^{5}$, qui est un composant essentiel de la vie humaine. Les normes, valeurs et pratiques éthiques sont en effet des composantes intrinsèques de tout environnement humain ${ }^{6}$, dont on ne voit guère pourquoi elles échapperaient à une définition du contexte ou de l'extralinguistique. Mais sur ce dernier paramètre, repose un soupçon d'idéologie, l'exclusion de l'axiologie ayant été justement un des supports de l'analyse linguistique scientifique ${ }^{7}$. La dimension morale des discours n'a donc pour cette raison jamais été prise en compte dans le champ des sciences du langage.

Quant à la dimension technologique ou technique de la production langagière et discursive, elle est sans doute mieux représentée dans les sciences du langage, mais sa prise en compte réelle dans l'analyse des données langagières reste rare : la dimension technologique est au mieux intégrée dans l'étude des supports, mais non dans l'étude des éléments langagiers euxmêmes. Or les discours nativement numériques, en particulier sur les réseaux sociaux, présentent des métissages technolangagiers dont il faut mener l'étude. Pour cela, la définition de l'objet langue admise en sciences du langage doit sans doute être un peu modifiée. La conception de la langue comme assemblage semble le permettre.

\section{La nature de la langue}

II est notable que les propositions les plus externalistes et les plus « symétriques » pour les données langagières ne sont pas dues prioritairement à des linguistes mais à des psychologues, comme Brassac et Sinha. Ces derniers avancent des hypothèses intéressantes pour une production langagière cognitivement distribuée dans les environnements humains et matériels, dans le cadre interactionniste.

J'ai parlé plus haut de Brassac, je détaille ici les propositions de Sinha, qui réclame en 2000 «[...] a serious theoretical-methodological encounter between cultural approaches to human development and cognitive linguistics needs to be put firmly on the agenda » (2000: 203). II met ensuite l'accent sur la nature cognitivo-communicationnelle du langage comme artefact issu de l'organisation socioculturelle de la vie humaine: "Natural language (I suggest) did not emerge, Athena-like, fully armed from the brow of an already-existent human 'Mind/Brain'. Rather, it emerged as a cognitive-communicative artefact on the basis of human sociocultural organization [...]» (2000: 204). Sa conclusion ouvre des perspectives fécondes pour l'analyse des données langagières : "In this view, there is no 'Mind/Brain', since 'mind' is co-constituted by the biological and social » (2000: 206). Dans un article ultérieur de 2005, il insiste sur le rôle des artefacts matériels dans la production langagière, ce qui permet de penser l'intégration de la dimension technologique à un programme linguistique :

[...] we argue [...] that the key notion of "embodiment" need[s] to be extended beyond its focus on the humanly corporeal, the "brain-in-the-body", to take account of the way that cognitive and cultural schemas find material realization - are embodied - in the artefacts of material culture; and the way in which such artefacts are themselves embedded in culturally appropriate, normative structures of action and interaction (Sinha 2005 : 1538).

Brassac tient des propos analogues, présentant l'interaction verbale comme une " production cognitive conjointe »: «Production cognitive conjointe, la construction de significations qui trame l'interaction entre sujets humains est une dynamique de modelage de formes langagières, corporelles et artefactuelles » (Brassac 2004 : 251-252). Dans un travail ultérieur, il considère que : «La création de matérialité est au cœur du dispositif intercognitif que constitue le déploiement de l'interaction » (Brassac 2007 : 12).

\footnotetext{
${ }^{5}$ Conformément aux usages en philosophie morale et philosophie de la connaissance, j'emploie ici éthique et morale comme synonymes.

${ }^{6}$ Et non humain, pourrait-on ajouter, si l'on pense aux travaux récents en philosophie et en éthologie sur l'existence d'une protomorale et d'un protolangage chez les animaux. Je ne développe pas ce point dans le cadre de cet article, mais je renvoie aux travaux de Lestel et Chapouthier en éthologie, Proust et Baumard en philosophie cognitive.

7 Je développe ce point dans Paveau 2010, 2012c et 2012d.
} 
Dans ce type d'approche, le langagier-discursif est un assemblage, c'est-à-dire une matière mixte, comportant du phonétique ou scriptural, du sémantique, du discursif, mais aussi du technologique : le non-langagier est dans le langagier, qui est un assemblage composite entre des «matières » diverses, dont la matière langagière classiquement postulée par la définition saussurienne du signe linguistique. Cela veut dire par exemple que les discours en ligne ne sont pas que des discours : ils sont aussi des technologies, des technologies du discours produisant des formes technolangagières.

\section{Du discours comme matériau composite}

J'aborde maintenant ces deux dimensions qui transforment les données langagières et discursives en matérialités composites, la dimension technologique et la dimension morale. II s'agit ici, dans le cadre restreint de cet article, plus d'esquisses que d'analyses détaillées ${ }^{8}$.

\subsection{Les discours en ligne comme technodiscours}

La notion de technologie discursive

La définition du langagier ainsi étendue à ses ancrages matériels et plus particulièrement technologiques oblige le linguiste à modifier ou à rendre mobiles ses corpus, ses objets, mais également ses théories. Dans le cadre de l'approche symétrique exposée plus haut, je propose la notion de "technologie discursive » (Paveau 2006, 2007, 2011) conçue comme un dispositif au sein duquel la production langagière et discursive est intrinsèquement liée à des outils technologiques (appareils, logiciels, applications, plateformes). Le terme technologie discursive s'inscrit dans le paradigme des composés de technologie, qui débute bien sûr avec technologie intellectuelle, qui apparait dans les années 1970. On trouve également technologie relationnelle (à partir de la fin des années 2000 chez Stiegler et l'ensemble des chercheurs autour du groupe Ars industrialis), technologie du langage chez Véronis, technologie du sexe ou technosexe chez Preciado. J'ai rencontré pour ma part la technologie intellectuelle sous la forme des «techniques matérielles et intellectuelles du travail intellectuel » il y a une quinzaine d'années chez Bourdieu et Passeron, dans une note du chapitre 2 de La reproduction, intitulé "Tradition lettrée et conservation sociale », dans la section «Langage et rapport au langage ${ }^{9}$. L'expression a un autre cheminement sans doute parallèle : apparu dans Bell 1973, le terme de technologie intellectuelle est diffusé le plus largement à partir de Goody 1977 et connait une fortune importante en sciences de l'information et de la communication, et en particulier chez les chercheurs qui s'occupent de l'internet et des dispositifs numériques. À partir de technologie discursive, je construis pour les besoins de mon analyse technodiscursif, technodiscours et technolangagier.

Des matérialités technolangagières et technodiscursives

Les productions natives en ligne, c'est-à-dire directement composées en ligne et non issues de la numérisation de productions imprimées traditionnelles ${ }^{10}$ ou de la production sur clavier téléphonique ${ }^{11}$, présentent des traits que j'appelle technolangagiers. Un trait technolangagier est une caractéristique de nature composite due à la production du langage dans un dispositif d'écriture numérique. On peut citer bien évidemment l'hyperlien, qui est un mot ou un segment technolangagier dans la mesure où il est cliquable et support d'hypertexte, le hashtag sur le réseau Twitter (Paveau 2012b), qui est un technomorphème dans la mesure où il permet par clic

\footnotetext{
${ }^{8}$ La proposition d'une éthique des vertus discursives fait l'objet d'un ouvrage à paraitre (Paveau 2012d) et les technologies discursives, pour l'instant exposées à l'oral et présentées en ligne (Paveau 2011, 2012 a et b) constituent l'objet d'un ouvrage en cours de rédaction : http://technodiscours.hypotheses.org/

${ }^{9}$ Rien d'étonnant si les étudiants que leur origine sociale prédispose à la désinvolture distinguée trahissent dans tant de leurs conduites le mépris aristocratique des besognes subalternes (reflet de l'opposition universitaire entre l'acte intellectuel parfaitement accompli et les démarches laborieuses du travail pédagogique), puisque l'institution scolaire relègue objectivement au dernier rang de sa hiérarchie l'inculcation méthodique des techniques matérielles et intellectuelles du travail intellectuel et du rapport technique à ces techniques (Bourdieu, Passeron 1970 : 153, note 21) .

${ }^{10}$ II faudrait être plus nuancé et envisager que la production imprimée traditionnelle, une fois numérisée, change de nature puisqu'elle devient entre autres, navigable et cliquable. II y a donc aussi bien évidemment du « techno » dans le numérisé.

${ }^{11}$ Il existe des travaux désormais assez nombreux, en CMO (Communication médiée par ordinteur) notamment, sur les écritures SMS, essentiellement sur téléphone et dans les forums (par exemple Liénard, Marcoccia, Panckhurst). Peu de travaux sont menés sur les écritures natives en ligne, qui dépassent les traits morphographiques habituellement traités (abréviations, smileys, etc.).
} 
de redocumenter un thème ou une information, ou encore les boutons de partage d'information, qui sont des formes technodiscursives de discours rapportés (Paveau 2011, 2012a). Certains genres de discours sont des genres technodiscursifs : la demande d'amitié sur Facebook, parce qu'elle passe nécessairement par le bouton «ajouter », et qu'elle peut de ce fait se passer d'un discours «traditionnel » (un message accompagnant la demande) constitue un genre technodiscursif.

La «boite à outil » des sciences du langage et de la théorie du discours n'est pas forcément équipée pour rendre compte de ce type de matérialités. L'écriture numérique ne peut plus être attribuée à un énonciateur ou scripteur conçu comme une instance unique : l'écriture en ligne est collaborative voire collective, par augmentation du sujet scripteur (les commentaires laissés par les internautes augmentent les textes, billets ou messages des sites et blogs), par écriture collective (des plateformes dédiées permettent une écriture collective) ou par reprise des textes selon différents formats (partage, reblogging, signalement par liens). Dans tous le cas, l'écriture en ligne relève plus de la poly-énonciation que de l'énonciation, l'intelligence y est collective et l'ubiquité naturelle. Du coup, une notion comme l'hétérogénéité énonciative, qui a été si féconde en théorie du discours, pourrait être repensée : la présence de l'autre dans le discours est diffractée et démultipliée, et les marqueurs linguistiques n'en sont plus forcément les indices. De même pour les outils descripteurs de l'analyse conversationnelle à l'écrit : la notion de tour de parole et ses marques sont modifiées sur un réseau comme Twitter, où l'on participe couramment à plusieurs conversations en même temps, et où les marques, en particulier ponctuatives, sont remplacées par des traits technodiscursifs (par exemple: mention du pseudo du destinataire pour une adresse). Le code lui-même est affecté : si les communications en ligne relèvent bien pour moi de l'écrit, celui-ci est modifié à la fois sur le plan des formes graphiques et des gestes scripturaux.

\subsection{L'hypothèse d'une vertu discursive?}

J'ai signalé plus haut que la dimension morale des énoncés pouvait être prise en compte comme paramètre contextuel au même titre que leur dimension sociale, historique, culturelle, etc. J'ajoute que certains travaux récents sur la naturalisation de la morale, qui reprennent la notion de sentiment moral très développée par la philosophie, en particulier anglaise, au $18^{\mathrm{e}}$ siècle, nous poussent intégrer le paramètre moral au contexte de toute activité humaine, dont la production des discours (Virvidakis 1994, Ogien (dir.) 1999, Baumard 2010). Enfin, l'ouverture de la philosophie de la connaissance à l'éthique, qui produit l'hypothèse d'une norme cognitive, c'est-à-dire l'idée qu'une connaissance vraie serait également bonne (Engel \& Mulligan, Engel, Tiercelin 2011), donne aussi des arguments pour un décloisonnement des catégories et pour leur ouverture à l'éthique.

\section{Des évènements discursifs moraux}

La question est bien sûr de savoir comment intégrer l'éthique à la théorie linguistique. Les questions sont les suivantes: $\mathrm{Y}$ a-t-il une raison et une explication de nature linguistique à l'indignation morale, parfois suivie de sanctions juridiques, que ces énoncés suscitent ? Autrement dit : peut-on poser la question morale à la théorie du discours ? Si notre équipement théorique et méthodologique est léger pour aborder la question des matérialités technodiscursives, il est en revanche inexistant pour traiter la question morale. On peut partir des données empiriques : les évènements discursifs autour de paroles publiques considérées comme moralement et/ou juridiquement contestables abondent dans la sphère médiatique, ce qui montre qu'il existe un " ordre du discours » touchant la question morale et/ou juridique. Il existe des énoncés que l'on ne peut pas produire et diffuser sans sanction, juridique ou sociale, et qui produisent ce que j'appelle des évènements discursifs moraux: ils produisent des métadiscours d'indignation morale, qui constituent une sorte d'éthique du discours spontanée, sur laquelle le chercheur peut s'appuyer pour observer les normes du discours telles qu'elles sont intersubjectivement négociées par les agents dans une société donnée ${ }^{12}$

Soit l'exemple suivant : en juillet 2008 éclate un scandale médiatique doublé d'une bataille discursive autour de l'emploi du mot rat par A. Badiou dans De quoi Sarkozy est-il le nom ? (2007,

\footnotetext{
12 II faut insister sur ce point : une éthique du discours ne doit pas être un corps de jugements d'acceptabilité éthique sur des énoncés, parce que la nature même des sciences du langage comme discipline non normative ne le lui permet tout simplement pas. Elle peut en revanche constituer un dispositif descriptif qui, à partir de métadiscours moraux, s'ouvre vers une configuration théorique.
} 
Paris, Lignes). Le débat remonte en fait à novembre 2007, date à laquelle P. Assouline, sur son blog La république des livres, publie un billet extrêmement critique sur le séminaire dont est issu l'ouvrage, sous le titre « De quoi Badiou est-il le nom ? » :

Au cours de son séminaire consacré en grande partie à la campagne électorale, un professeur de philosophie dont Louis Althusser fut le maitre, Alain Badiou [...] a longuement parlé du candidat Nicolas Sarkozy en le désignant essentiellement, bien après Freud mais sans rapport avec le cas célèbre, comme "l'homme aux rats" et de ses électeurs comme des "rats". Les fidèles de ce blog savent que je ne suis pas soupçonnable de complaisance envers le personnage, mais un Rubicon est franchi dans cet avilissement, d'autant qu'il se manifeste du haut d'un magistère au sein d'une fabrique à élites. La dernière fois dans ce pays qu'on a ainsi comparé des hommes à des rats, c'était, voyons, en 1942 dans un documentaire de propagande sur le péril juif (28.11.2007, http://passouline.blog.lemonde.fr/2007/11/, extrait).

Ce billet (qui reçoit 487 commentaires, chiffre assez élevé par rapport à la moyenne des blogs, même célèbres), relayé dans la presse et dans les milieux intellectuels, provoque une polémique autour de la sortie du livre. II porte sur ce passage (je souligne les occurrences de rat) :

Si vous êtes prisonniers de la temporalité d'opinion, vous allez vous dire, comme tant de caciques ou d'électeurs socialistes, « Nom de Dieu ! On subissait Chirac depuis douze ans, et maintenant il va falloir attendre le prochain tour ! Dix-sept ans ! Peut-être vingt-deux ! une vie entière ! Ce n'est pas possible ! » Et alors, au mieux vous êtes déprimé, au pire vous devenez un rat. Le rat est celui qui, interne à la temporalité d'opinion, ne peut supporter d'attendre. Le prochain tour commandé par l'Etat, c'est très loin. Je vieillis, se dit le rat. Lui, il ne veut pas mariner dans l'impuissance, mais encore moins dans l'impossible ! L'impossible, très peu pour lui. II faut reconnaitre à Sarkozy une profonde connaissance de la subjectivité des rats. Il les attire avec virtuosité. Peut-être a-t-il été rat lui-même ? En 1995, quand, trop pressé, d'en venir aux choses ministérielles sérieuses, il a trahi Chirac pour Balladur ? En tout cas, trouvant les usages d'Etat de la psychologie du rat, il mérite un nom psychanalytiquement fameux. Je propose de nommer Nicolas Sarkozy "l'homme aux rats ». Oui, c'est juste, c'est mérité. Le rat est celui qui a besoin de se précipiter dans la durée qu'on lui offre, sans être du tout en état de construire une autre durée. Le point à trouver doit être tel qu'on puisse lui annexer une durée différente. N'être ni rat ni déprimé, c'est construire un temps autre que celui auquel l'Etat, ou l'état de la situation, nous assigne. Donc un temps impossible, mais qui sera notre temps (Badiou 2007 : 47).

La polémique rebondit en juillet 2008, quand B.-H. Lévy publie un article de soutien à $P$. Val ${ }^{13}$ dans Le Monde, article qui reprend les critiques de $\mathrm{P}$. Assouline en ajoutant la dimension antisémite :

Dans un livre récent, De quoi Sarkozy est-il le nom ?, Alain Badiou s'autorisait de sa juste lutte contre l'« immonde » pour réintroduire dans le lexique politique des métaphores zoologiques (« les rats »..., " l'homme aux rats ») dont le Sartre de la préface aux Damnés de la terre avait pourtant démontré, sans appel, qu'elles sont toujours la marque du fascisme (Le Monde, 22.07.2008).

A. Badiou répond dans le même quotidien le 25 juillet, dans un article intitulé «Tout antisarkozyste est-il un chien ? » :

M. Assouline a remarqué, sur son blog, que je traitais de " rats " les socialistes entrés au gouvernement Sarkozy, et, par voie de conséquence, Sarkozy lui-même d'« homme aux rats". Quelqu'un de très modérément cultivé sait aussitôt que j'entrelace ici, non sans une subtilité rhétorique qui mériterait des éloges, la métaphore des rats qui quittent le navire, la légende du joueur de flite qui entraine les rats hors de la ville, et le cas, décrit par Freud, de " l'homme aux rats » comme exemple type de l'obsession. M. Assouline est-il cultivé ? II sait en tout cas où il veut en venir. Depuis la dernière guerre et les nazis (suivez mon regard) proclame-t-il, personne n'a plus traité qui que ce soit de rat. Par ailleurs il y a des juifs dans la généalogie de Sarkozy. Donc... vous voyez? Hein ? Vous voyez bien ? [...] On ne peut pas toujours comparer les gens à des aigles, comme on l'a fait pour Bossuet, ni même à des bœufs, comme ce fut le cas pour le président du conseil Joseph Laniel, ou encore à des renards, comme c'était courant s'agissant de Mitterrand (A. Badiou, Le Monde, 25.07.2008).

La vertu discursive : décence, vérité, mémoire

\footnotetext{
${ }^{13}$ Celui-ci qui vient de renvoyer Siné de Charlie Hebdo, à la suite d'une chronique sur le fils de N. Sarkozy, considérée comme antisémite.
} 
À partir de l'analyse de nombreux évènements discursifs de ce type (Paveau 2010, 2012c, 2012d), j'ai proposé de mettre en place la notion de vertu discursive définie comme une disposition des agents-locuteurs (énonciateur et co-énonciateur dans un même processus) à produire des énoncés ajustés aux valeurs admises qui sous-tendent, dans un état donné de l'histoire d'une société selon les trois axes suivants : un ajustement à la décence discursive ${ }^{14}$ concernant les relations entre les agents ; un ajustement à la vérité du monde concernant la manière de dire l'état du monde; un ajustement à la mémoire discursive dans laquelle se tissent les discours d'une société.

La critique d'Assouline et de BHL porte sur l'usage du mot rat comme désignatif de l'électeur de N. Sarkozy, usage produisant des effets d'indignation morale (traduite par le terme d'avilissement chez Assouline et l'accusation de "fascisme » portée par BHL), qui prennent leur source dans des inajustements enchevêtrés à des mémoires discursives. La description de ces inajustements permet peut-être de comprendre les effets idéologiques et moraux (les deux étant mêlés) de cet emploi, et de montrer que les usages de ce mot, passés, présents et futurs, sont désormais marqués de cette dimension.

Premier inajustement : l'animalisation. Elle est considérée comme inacceptable par les détracteurs du philosophe, même si, dans le texte de Badiou, on peut apercevoir les traces d'une mémoire littéraire, celle des Fables de La Fontaine par exemple. Mais cette mémoire du genre de la fable n'est sans doute pas suffisamment présente dans le texte de Badiou pour que l'effet-fable dépasse l'effet-animalisation. De même pour l'allusion au joueur de flite, légère mais présente (« il les attire avec virtuosité »), que Badiou rappelle didactiquement dans sa défense. Cette animalisation est d'autant plus inacceptable pour certains récepteurs que l'animal choisi porte de lourdes valeurs axiologiques négatives, assez peu présentes dans les dictionnaires, curieusement, mais bien identifiables dans les prédiscours verbaux et iconiques (romans, films, bandes dessinées, où les aspects sale, grouillant, destructeur et grégaire du rat sont largement exploités).

Deuxième inajustement : la mémoire du discours nazi. Une mémoire discursive plus spécifique, celle de l'animalisation langagière et iconique des juifs et des catégories pourchassées et exterminées par le régime nazi semble en effet venir construire l'interprétation, d'autant plus autorisée qu'un précédent ouvrage de Badiou (2005, Portées du mot « juif », Paris, Lignes) est déjà au cœur d'une polémique. Cette mémoire de l'animalisation, soutenue par la référence à Sartre, est peut-être aussi (mais ici c'est ma propre mémoire discursive que je mobilise) rendue encore plus saillante par la bande dessinée d'A. Spiegelman, Maus, particulièrement connue et traduite dans le monde entier, où les juifs sont représentés en souris.

Troisième inajustement: la mémoire discursive freudienne et lacanienne. Tout le texte de Badiou est imprégné de références à la psychanalyse, à travers le cas de l'homme aux rats, qui est l'une des études cliniques les plus célèbres de Freud, mais également dans l'allusion à Lacan que constitue le jeu sur les mots impuissance et impossible. Il se trouve que cette mémoire-là n'est pas du tout mentionnée par ses détracteurs et le discours de Badiou apparait donc inajusté à son auditoire. La psychanalyse est effacée de la polémique, alors qu'elle aurait pu être une clé interprétative garantissant un ajustement, et les commentateurs du texte procèdent à une « remémorisation » en suivant d'autres lignées discursives. Cela mène Badiou, dans sa réponse, à une explication de texte un peu scolaire qui n'aurait peut-être pas dû être nécessaire, si le texte avait été écrit de manière plus réflexive, c'est-à-dire en mesurant ses effets possibles, par le biais d'un calcul interprétatif.

C'est donc l'environnement cognitif et culturel du texte, et les réactions qu'il suscite, qui signalent un discours problématique, non conforme aux normes éthiques partagées, et non l'emploi du mot en soi. L'évènement discursif provoqué par cet emploi est dépendant des environnements énonciatifs et interprétatifs, qui s'appuient sur des potentialités de sens du mot, et sur son histoire sémantique.

\section{Conclusion}

\footnotetext{
${ }^{14}$ Je propose cette notion à partir de l'usage qu'en fait Margalit dans La société décente : « [...] une société décente est une société dont les institutions n'humilient pas les gens. Je fais la distinction entre une société décente et une société civilisée. Une société civilisée est celle dont les membres ne s'humilient pas les uns les autres, alors qu'une société décente est celle où les institutions n'humilient pas les gens » (Margalit 1999 [1996] : 13).
} 
Analyser l'environnement comme constitutif des productions verbales suppose de modifier un peu nos théories et méthodes. Si nos objets deviennent composites, comme les matérialités technolangagières des discours en ligne, et si le contexte n'est plus réduit au " socio-historique " mais étendu à l'ensemble des dimensions de la vie humaine, alors le langagier n'est peut-être plus aussi facilement définissable et observable comme objet homogène.

II est en effet probable que les renouvèlements postdualistes de l'ensemble des sciences humaines et sociales aient, à plus ou moins long terme, des effets sur la théorie du discours.

\section{Bibliographie}

N.B. tous les liens ont été consultés et vérifiés le 21/04/2012.

Achard-Bayle G., (éd.), 2006, Pratiques 129-130, « Textes. Contextes ».

Achard-Bayle G., Paveau, M.-A.

- (eds.), 2007, “Contextes, discours, cognitions », numéro spécial de la revue Corela (Cognition, Représentation, langage) http://corela.edel.univ-poitiers.fr/sommaire.php?id=1559.

- 2012, (à par.), "Réel, contexte, et cognition. Contribution à une histoire de la linguistique cognitive ", Histoire Épistémologie Langage XXXIV (1).

Atelier de réflexion prospective "Sciences et Technologies Cognitives », janvier 2010, Rapport de synthèse de l'atelier PIRSTEC, http://pirstec.risc.cnrs.fr/

Auroux S., 1998, La raison, le langage et les normes, Paris, PUF.

Bauman Z.,

- 2010 [2004], Identité, trad. M. Dennehy, Paris, L'Herne.

- 2007, Le présent liquide. Peurs sociales et obsessions sécuritaires, trad. L. Bury, Paris, Seuil.

Baumard N., 2010, Comment nous sommes devenus moraux. Une histoire naturelle de la morale, Paris, Odile Jacob.

Bell D., 1976 [1973], La société post-industrielle, trad. P. Andler, Paris, Robert Laffont.

Berrendonner A., 1981, Éléments de pragmatique linguistique, Paris, Minuit.

Bischofsberger M., 2002, "Quel constructivisme pour la linguistique cognitive ? », in Bouquet S., Rastier F. (dir.), Introduction aux sciences de la culture, Paris, PUF, 157-176.

Bourdieu P., Passeron J.-C., 1970, La reproduction. Éléments pour une théorie du système d'enseignement, Paris, Les Éditions de Minuit.

Brassac C.

- 2004, "Action située et distribuée et analyse du discours: quelques interrogations ", Cahiers de linguistique française 26, 251-268.

- (dir.), 2006, «Internalisme / Externalisme », Intellectica 43.

- 2007, Co-responsabilité cognitive et dissolution de frontières. In P. Hert, M. Paul-Cavallier (éds), Sciences et frontières. Délimitations du savoir, objets et passages. Fernelmont (BE) : Éditions Modulaires Européennes \& InterCommunications, 159-176.

- et al. 2008a, "Interweaving objects, gestures, and talk in context », Mind, Culture and Activity: An International Journal, 15(2), 208-233.

- et al., 2008b, "Analyse de l'émergence de connaissances au cours d' un processus collectif. Recatégorisations, reformulations, stabilisations ", Revue d’ Anthropologie des Connaissances, Vol. 2, n², 267-286.

Chapouthier G., Kaplan F. 2011, L'homme, l'animal et la machine, Paris, CNRS Éditions.

Clark A., 1997, Being There : Putting Brain, Body, and World Together Again, Cambridge (MA), MIT Press.

Clark A., Chalmers D., 1998, « The extended mind », Analysis 58 (1), 10-23.

Damasio A., 2005 [2003], Spinoza avait raison. Joie et tristesse, le cerveau des émotions. Paris, Odile Jacob.

Dennett D.C., 1993 [1991], La conscience expliquée, trad. P. Engel, Paris, Odile Jacob.

Engel P., 2012, Épistémologie pour une marquise, Paris, Ithaque.

Engel P., Mulligan K., 2003, « Normes éthiques et normes cognitives », Cités 2003/3, n 15, 171-186.

Goody J., 1979 [1977], La raison graphique. La domestication de la pensée sauvage, trad. J. Bazin, Paris, Minuit.

Honneth A., 2008 [1993], « L'autonomie décentrée. Les conséquences de la critique moderne du sujet pour la philosophie morale ", dans Psychologie morale, Autonomie, responsabilité et rationalité pratique, Paris, Vrin, 347-364.

Hutchins $\mathrm{E}$.

- 1994 [1991], "Comment le cockpit se souvient de ses vitesses », Sociologie du travail 4 : 461-473.

- 1995, Cognition in the Wild, Bradford Books-MIT Press, Cambridge MA.

Lahlou S. 
- 2000a, «La cognition au travail et ses outils : débordement, révolution, distribution », Intellectica 30, 7-17.

- 2000b, «Attracteurs cognitifs et travail de bureau », Intellectica 30, 75-113.

Laplantine F., 2010 [1999], Je, nous et les autres, Paris, Le pommier.

Latour B.

- 1994, « Une sociologie sans objet ? remarque sur l'interobjectivité », Sociologie du travail 4, 587-606.

- 1997, Nous n'avons jamais été modernes. Essai d'anthropologie symétrique, Paris, La Découverte.

- 2005, Reassembling the social: an introduction to Actor-Network-Theory, New York, Oxford University Press.

Lenay C., 2006, « Enaction. Externalisme et suppléance perceptive », Intellectica 43, 27-52.

Margalit A., 1999 [1996], La société décente, Paris, Climats.

Ogien R. (dir.), 1999, Le réalisme moral, Paris, PUF.

Paveau M.-A.

- 2006, Les prédiscours. Sens, mémoire, cognition, Paris, Presses Sorbonne nouvelle.

- 2007, "Discours et cognition. Les prédiscours entre cadres internes et environnement extérieur », Corela : http://corela.edel.univ-poitiers.fr/document.php?id=1672

- 2009, "Mais où est donc le sens ? Pour une linguistique symétrique ", conférence invitée au deuxième colloque international Res per nomen, Reims, 30-31 mai, in Actes prépubliés, 21-31.

- 2010, "Vices et vertus du discours universitaire. Une perspective éthique ", dans Les discours universitaires : formes, pratiques, mutations, actes du colloque international, Bruxelles, avril 2008, Paris, L'Harmattan, 111-124.

- 2011, "Les technologies discursives. Altérisation, cognition, narcissisme », intervention au Séminaire COSTECH consacré aux « Altérités technologiques », 21 janvier.

- 2012a, "Les amitiés numériques. Formes technolangagières et polysémie relationnelle ", La pensée du discours, http://penseedudiscours. hypotheses.org/? $\mathrm{p}=8334$

- 2012b, "Activités langagières et technologie discursive. L'exemple de Twitter ", La pensée du discours, 27.02.2012, http://penseedudiscours. hypotheses.org/8338

- 2012c (à par.) : "Théorie du discours et philosophie morale ", Actes du colloque international Le rapport éthique au discours (Université de Montpellier 3, avril 2011), Berne, Peter lang.

- 2012d (à par.), Langage et morale. Une éthique des vertus discursives, Lyon, ENS Éditions.

Pêcheux M., Haroche C., Henry P., 1971, "La sémantique et la coupure saussurienne ", Langages 24, "Épistémologie de la linguistique », in Maldidier D., L'inquiétude du discours, Paris, Éditions des cendres, 133-153.

Plantin, C., 2011, Les bonnes raisons des émotions. Principes et méthode pour l'étude du discours émotionné, Berne, Peter Lang.

Putnam $\mathrm{H}$.

- 1994 [1990], Le Réalisme à visage humain, trad. Claudine Tiercelin-Engel, Paris, Seuil.

- 1999, The Threefold Cord: Mind, Body, and World, New York, Columbia University Press.

- 2004 [2002], Fait/Valeur: la fin d'un dogme et autres essais, trad. M. Caveribère et J.-P. Cometti, ParisTel-Aviv, Éditions de L'éclat.

Schaeffer J.-M., 2007, La fin de l'exception humaine, Paris, Gallimard.

Sinha C.

-2000, "Culture, Language and the Emergence of Subjectivity », Culture \& Psychology 6 (2), 197-207.

- 2008, "Blending out of the background: Play, props and staging in the material world ", Journal of Pragmatics 37, 1537-1554.

Tiercelin C., 2011, Le ciment des choses. Petit traité de réalisme métaphysique, Paris, Ithaque.

Virvidakis S., 1994, La robustesse du bien. Essai sur le réalisme moral, Nimes, Jacqueline Chambon. 